University of Nebraska - Lincoln

DigitalCommons@University of Nebraska - Lincoln

Gordon Gallup Publications

Research Papers in Physics and Astronomy

December 1952

\title{
A Theoretical Formulation of the Hammett Equation
}

Gordon A. Gallup

UNL,ggallup1@unl.edu

W.R. Gilkerson

University of Kansas - Lawrence

M.M. Jones

University of Kansas - Lawrence

Follow this and additional works at: https://digitalcommons.unl.edu/physicsgallup

Part of the Physics Commons

Gallup, Gordon A.; Gilkerson, W.R.; and Jones, M.M., "A Theoretical Formulation of the Hammett Equation" (1952). Gordon Gallup Publications. 32.

https://digitalcommons.unl.edu/physicsgallup/32

This Article is brought to you for free and open access by the Research Papers in Physics and Astronomy at DigitalCommons@University of Nebraska - Lincoln. It has been accepted for inclusion in Gordon Gallup Publications by an authorized administrator of DigitalCommons@University of Nebraska - Lincoln. 


\section{A Theoretical Formulation of the Hammett Equation}

\section{G. A. GALLUP*. W. R. GILKERSON* and M. M. JONES**} University of Kansas, Lawrence

One of the aims of physical chemistry is to explain and correlate the empirical facts of chemistry. One of the more useful generalizations of organic chemistry is the Hammett equation. ${ }^{1}$ This equation correlates the rates of over two hundred reactions with the structure of the reactants. The equation as it stands now is applicable only to reactions of aromatic compounds and their derivatives. The Hammett equation is usually written,

$$
\log _{10} \frac{k}{k_{0}}=p \sigma
$$

$\mathbf{k}_{\mathbf{0}}$ is the specific reaction rate constant for a given reaction with an unsubstituted reactant, $\mathrm{k}$ is the rate constant of the reaction using a substituted reactant, $\rho$ is a constant determined by the reaction, and $\sigma$ is a constant determined by the substituent. For example consider the two reactions:
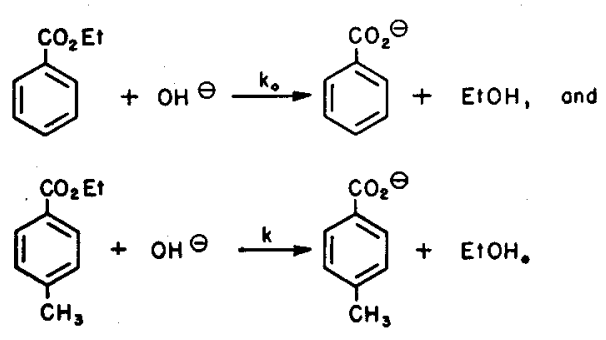

For these two reactions,

$$
\log _{10} \frac{k}{k_{0}}=\rho \sigma=(2.498)(-0.170)=-0.424
$$

$\rho$ for this reaction is 2.498

$\sigma$ for the methyl group is -0.170

Experimental data may be fitted to this equation with a deviation of $\pm 15 \%$.

In our formulation we have considered two types of idealized reactions. In each of these cases the aromatic compound is considered to be a dipole essentially and the attacking reagent a point charge or another dipole depending on the type of reaction. We have assumed that the energy of activation can be split into two terms, $E_{A}=E_{0}+E_{e}$.

Transactions of the Kansas Academy of Science, Vol. 55, No. 2, 1952.

*AEC Predoctoral Fellows

1 Hammett, Chem. Rev. 17, 125 (1935) where $\mathrm{E}_{0}$ is that part of the activation energy due to bond breaking and formation, and $\mathrm{E}_{\mathrm{e}}$ is the electrical interaction energy of the reactant molecules due either to charge or to existence of permanent dipoles. We have assumed $\mathrm{E}_{\mathrm{o}}$ to be dependent only on the reaction and specifically to be independent of the substituent. In each of our special cases we have assumed a specific functional form for $\mathrm{E}_{\mathrm{e}}$, and we have further assumed that the rate determining step is the approach of the attacking ion or dipole to the aromatic molecule.

I. Ion-dipole reactions

In this case the electrical energy is given $b y^{2}$,

$$
\frac{E_{e}}{N_{0}}=\frac{Z_{e} \mu \cos \theta}{D r^{2}}
$$

where $Z_{e}$ is the charge of the attacking ion,

$\mu$ is the dipole moment of the attacked molecule,

$\theta$ is the angle of orientation of the ion to the dipole,

$\mathrm{D}$ is the dielectric constant of the medium,

$r$ is the linear separation of $Z_{e}$ and $\mu$, and

$\mathbf{N}_{\mathrm{o}}$ is Avogadro's Number.

From the theory of absolute reaction rates,

$$
k=\mathcal{H} \frac{k T}{h} \text { e } \frac{\Delta S^{\ddagger}}{R} e-\frac{E_{A}}{R T}
$$

where $\mathrm{k}$ is the specific reaction rate constant,

$x$ is the transmission coefficient,

$\kappa$ is the Boltzmann constant,

$\mathrm{T}$ is the absolute temperature,

$\mathrm{h}$ is the Planck constant,

$R$ is the gas constant, and

$\Delta \mathrm{S}^{\ddagger}$ is the entropy of activation.

In our development we have assumed that $x$ and $\Delta S^{\ddagger}$ depend only on the reaction and are independent of the substituent.

Making the proper substitutions,

$$
k_{0}=H \frac{k T}{h} \text { e } \frac{\Delta S^{\ddagger}}{R} e^{-} \frac{E_{A}^{(0)}}{R T} \text {, and } k=H \frac{k T}{h} \text { e } \frac{\Delta S^{\ddagger}}{R} \text { e- } \frac{E_{A}}{R T}
$$

Dividing and taking logarithms, $\log _{10} \frac{k}{k_{0}}=\frac{E_{A}^{(0)}-E_{A}}{2.303 R T}$

$$
=\frac{Z_{e} \operatorname{Cos} \theta}{2.303 D r^{2} k T}\left(\mu_{0}-\mu\right)
$$

2 Moelwyn-Hughes, "Kinetics of Reactions in Solutions", Oxford, 2nd Ed. (1947) p. 145 
By making the proper division of the factors, we may write,

$$
\begin{aligned}
\log _{10} \frac{k}{k_{0}} & =\rho^{\prime} \sigma^{\prime} \\
\rho^{\prime} & =\frac{Z_{e}}{2.303 D r^{2} k T} \\
\sigma^{\prime} & =\left(\mu_{0}-\mu\right) \cos \theta
\end{aligned}
$$

The simplification of the first expression is made possible by the assumptions that $\mathrm{r}, \theta$, and $\mathrm{D}$ are not changed by the substituent. Our $\boldsymbol{\rho}^{\prime}$ and $\sigma^{\prime}$ should be only proportional to Hammett's $\rho$ and $\sigma$, but the respective products should be equal.

\section{Dipole-dipole reactions}

In this case the electrical energy is given by ${ }^{3}$,

$$
\frac{E_{e}}{N_{0}}=\frac{\mu_{A} \mu_{B}}{D r^{3}} f\left(\theta_{A}, \theta_{B}\right)
$$

where $\mu_{\mathrm{A}}$ and $\mu_{\mathrm{B}}$ are the dipole moments of the attacked and the attacking molecules, respectively, $\mathrm{r}$ is the distance of separation of the two dipoles, and $\mathrm{f}\left(\theta_{\mathrm{A}}, \theta_{\mathrm{B}}\right)$ is a function of the two angles of orientation of the two dipoles. In most cases $\mathrm{f}\left(\theta_{\mathrm{A}}, \theta_{\mathrm{B}}\right)$ can be assumed to have an absolute value of 1.4

Using the same assumptions concerning $\varkappa, \Delta S^{\ddagger}, E^{o}, D, r$, and f $\left(\theta_{A}, \theta_{B}\right)$ constant, a similar treatment to I gives,

$$
\begin{aligned}
\log _{10} \frac{k}{k_{0}} & =\frac{\mu_{B}}{2.303 D k T_{r}^{3}}\left(\mu_{A_{0}}-\mu_{A}\right) f\left(\theta_{A}, \theta_{B}\right) \\
& =\rho^{\prime \prime} \sigma^{\prime \prime} \\
\rho^{\prime \prime} & =\frac{\mu_{B}}{2.303 D k T_{r}^{3}} \\
\sigma^{\prime \prime} & =\left(\mu_{A_{0}}-\mu_{A}\right) f\left(\theta_{A}, \theta_{B}\right) \cong \sigma^{\prime}
\end{aligned}
$$

From these treatments we may calculate a table of ratios of $\frac{\sigma^{\prime}}{\sigma}$ from values for group dipole moments as given in Gilman ${ }^{5}$, and values of $\sigma$ as given by Hammett ${ }^{6}$.

\begin{tabular}{|c|c|c|}
\hline \multirow{2}{*}{ Substifuent } & \multicolumn{2}{|c|}{$(\sigma / \sigma) \times 10^{10}$} \\
\hline & para & meto \\
\hline$-\mathrm{NO}_{2}$ & 5.18 & 4.41 \\
\hline - CHO & 2.45 & 3.61 \\
\hline$-\mathrm{CN}$ & 3.89 & 2.87 \\
\hline$-\mathrm{CH}_{3}$ & 2.65 & 3.26 \\
\hline$-\mathrm{NH}_{2}$ & 2.35 & 4.81 \\
\hline$-\mathrm{Cl}$ & 6.87 & 2.09 \\
\hline$-\mathrm{Br}$ & 6.55 & .1 .94 \\
\hline$-I$ & 4.60 & 1.80 \\
\hline$-\mathrm{OCH}_{3}$ & -3.75 & 5.20 \\
\hline
\end{tabular}

\footnotetext{
Idem. ibid, p. 205.

4 Idem, ibid. p. 213.

'H. Gilman, "Organic Chemistry", Wiley and Sons, 2nd Ed. N. Y. (1943) p. 1755.

'Hammett, "Physical Organic Chemistry", McGraw-Hill Book Co., N. Y. (1940) p. 188.
}

Inse 1

\begin{tabular}{|c|c|c|}
\hline \multicolumn{2}{|r|}{ Reoction } & \multirow{2}{*}{$\begin{array}{c}0^{\prime} 0^{\circ} \\
-3.02 \times 10^{18}\end{array}$} \\
\hline & $\begin{array}{l}\text { Alkoline hydrolysis of ethyl benzootes, } \\
87.83 \% \text { ethonol, } 30^{\circ} \mathrm{C} \text {. }\end{array}$ & \\
\hline & Alkaline hydrolysis of benzamides, water, $100^{\circ} \mathrm{C}$ & $-1.37 \times 10^{10}$ \\
\hline & Solvolysis of benzoyl chlorides, ethonol, $0^{\circ} \mathrm{C}$ & $-4.00 \times 10^{10}$ \\
\hline & Solvolysis of benzoyl chlorides, methanol, $0^{\circ} \mathrm{C}$ & $-3.12 \times 10^{10}$ \\
\hline
\end{tabular}

It must be emphasized that the Hammett equation represents the experimental data to within only $\pm 15 \%$. The deviation of the values in Table 1 is $\pm 30 \%$.

\begin{tabular}{|c|c|c|c|}
\hline Reaction & Group & $p \sigma$ & $\rho^{\prime} \sigma^{\prime}$ or $\rho^{\prime \prime} \sigma^{\prime}$ \\
\hline I. & & -0.424 & -0.453 \\
\hline 2. & $\mathrm{O}-\mathrm{CH}$ & -0.179 & -0.205 \\
\hline 3. & $2 \mathrm{Nin}_{3}$ & -0.260 & -0.522 \\
\hline 4. & & -0.249 & -0.488 \\
\hline
\end{tabular}

Table 2.

Table 2 gives values of $\rho^{\prime}$ for reactions 1 and 2 , and $\rho^{\prime \prime}$ for reactions 3 and 4 , assuming a value of $r=4.0 \mathrm{~A}$, which will be discussed later in this paper.

\section{Discussion}

The idealization we have introduced must be kept in mind when applying these formulae. The essential assumptions made have been:

1. The activation energy can be divided into two portions $\mathbf{E}_{\mathbf{o}}$ and $\mathbf{E}_{\mathrm{e}}$, each of which can be assigned to definite causes. The importance of this step is that it allows us to write a definite functional relation for $E_{e}$ in each 
of our two cases. The fact that we have assumed $\mathrm{E}_{0}$ constant for a given reaction allows us to obtain an expression similar in form to the Hammett. equation.

2. That the rate-determining step is the same for different substituents except for a term due solely to the ion-dipole or dipole-dipole interacions. This statement is tantamount to the assumption that the mechanisms follow essentially the same path.

3. That $x$ and $\Delta S^{\ddagger}$ for a given reaction are independent of the nature of the substituent. This assumption means that some of the reactions correlated by the Hammett equation, where $\Delta S^{\ddagger}$ does not remain constant, cannot be interpreted by this model.

4. The distance of closest approach is 4.0A. That this is a reasonable value may be seen by a consideration of the interatomic distances. in the benzene ring.

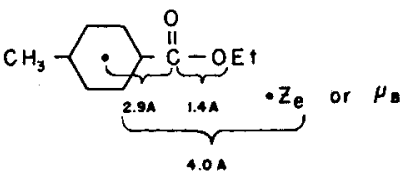

5. That the dielectric constant of the medium is to be considered as the dielectric constant of the region between the dipole in the aromatic ring and the ion or dipole. We feel that this is justified, since, in the calculation of the work required to bring the two reactants within $4.0 \mathrm{~A}$ of each other the major contribution is from the region where the dielectric constant is that of the medium.

6. That the function of $\theta$ in the dipole-dipole interaction energy is roughly that of Moelwyn-Hughes. Also, since the value of $\theta$ in the ion-dipole case is assumed to give a cosine of either + or -1 , it may be included in the $\sigma^{\prime}$ factor. This treatment removes the dependence of $\cos \theta^{\prime}$ on the reaction type.

\section{Summary}

A relation, which reduces to the form of Hammett's equation, has been derived. Reactions which can be assumed to be of the form iondipole or dipole-dipole have been considered. The logarithms of the ratios of rate constants were calculated and compared with experimental values in the form of Hammett's equation constants. 